\title{
Clinical and genetic features of pediatric PCCs/PGLs patients: a single-center experience in China
}

\author{
Minghao Li", Cikui Wang", Peihua Liu, Lin Qi, Xiang Chen, Benyi Fan, Xiangyang Zhang, Bo Zhang, \\ Qiao Xiao, Anze Yu, Longfei Liu \\ Department of Urology, Xiangya Hospital, Central South University, Changsha 410008, China \\ Contributions: (I) Conception and design: L Liu, M Li, C Wang; (II) Administrative support: L Qi, X Chen, B Fan, X Zhang; (III) Provision of \\ study materials or patients: L Qi, X Chen, B Fan, X Zhang; (IV) Collection and assembly of data: C Wang, M Li, Q Xiao; (V) Data analysis and \\ interpretation: P Liu, M Li, C Wang, A Yu, L Liu, B Zhang; (VI) Manuscript writing: All authors; (VII) Final approval of manuscript: All authors. \\ \#These authors contributed equally to this work. \\ Correspondence to: Longfei Liu, MD. Department of Urology, Xiangya Hospital, Central South University, No. 87 Xiangya Road, Changsha 410008, \\ China. Email: longfei_liu@csu.edu.cn.
}

\begin{abstract}
Background: Although $40 \%$ to $80 \%$ of pediatric patients with pheochromocytoma (PCC) and paraganglioma (PGL) have been reported to carry germline mutations, the genetic and clinical features are poorly understood, and few such patients have undergone genetic testing. In this series, we aimed to investigate the clinical and genetic features of Han Chinese pediatric patients with PCC/PGL.

Methods: The medical records of 15 pediatric patients with PCC/PGL who presented to our hospital between 2006 and 2018 were retrospectively studied. DNAs isolated from leukocytes of the patients were analyzed using whole-exome sequencing (WES).

Results: The patients were nine girls and six boys with a mean age of 14.9 (range, 6-18) years. All were alive after a follow-up from 1 to 12 years, although two were diagnosed with pulmonary metastatic PGLs. Four patients were diagnosed with bilateral PCCs. Four patients were diagnosed with tumor syndromes. Among the 15 patients, nine were identified carrying germline mutations, of which seven were $V H L$ and one each of RET and SDHB. In addition, a de novo mutation, $V H L$ c.193T >A, was identified in a patient clinically diagnosed with a $V H L$ syndrome.

Conclusions: Among 15 pediatric patients studied, nine were identified carrying germline genetic mutations, four were diagnosed with bilateral PCCs, and four were diagnosed with other syndromic tumors in addition to PCC, which underscores the importance of genetic testing and managing treatment accordingly.
\end{abstract}

Keywords: Paraganglioma (PGL); genetic counseling; pediatrics; pheochromocytoma (PCC); VHL mutation

Submitted Nov 04, 2019. Accepted for publication Feb 14, 2020.

doi: $10.21037 /$ tau.2020.02.14

View this article at: http://dx.doi.org/10.21037/tau.2020.02.14

\section{Introduction}

Pheochromocytomas (PCCs) and paragangliomas (PGLs) are neuroendocrine tumors derived from chromaffin cells of the adrenal medulla or extra-adrenal locations. The incidence of PCCs/PGLs in the pediatric population is 0.3 cases per million persons per year or less (1). Despite its low incidence, it is the most frequent endocrine tumor in children and accounts for $0.5-1 \%$ of pediatric hypertensive cases (2). Symptoms of this disease include headaches, sweating, and palpitations caused by the excessive secretion of catecholamines (1). PCCs/PGLs were originally called "the $10 \%$ tumor" because the proportion of bilateral occurrences, metastases, inheritance, and tumors presenting during childhood is $10 \%$. However, in recent years, more than $30 \%$ of PCCs/PGLs have been reported to have 
genetic mutations in the following genes: VHL, RET, $S D H x, N F 1, T M E M 127, M A X$, and FH (3). In addition to these genes, the following genes have also been reported to be related to the aetiology of PCCs/PGLs: EGLN1, KIF1B, EPAS1, MDH2, BRCA1, BRCA2, KMT2D, IDH, MEN1, and $A T R X$ (4). The proportion of patients with inherited genes is even higher in pediatric patients, with $40-80 \%$ reported of carrying germline mutations $(5,6)$.

The standard treatment for PCCs/PGLs is total adrenalectomy (7). However, for patients with hereditary disease, especially with mutations related to bilateral PCCs, adrenal-sparing surgery is recommended though which leaving the risk of recurrence and metastasis (8), this highlights the importance of genetic consulting. For pediatric patients, this is a peculiar question because $40-80 \%$ of these patients carry germline mutations (much higher than that of adults) and genetic mutations are related to bilateral morbidity and recurrence. Furthermore, germline mutations in genes, including VHL, RET, NF-1, and $S D H x$ are associated with tumor syndromes and metastatic PCCs/PGLs (9). Therefore, the detection of genetic mutations may profoundly influence clinical outcomes and disease management. However, in clinical pediatric cases, only a small minority of patients have undergone genetic testing (10-12). Since the data for pediatric PCCs/PGLs are very limited, the clinical management of PCCs/PGLs in children or adolescents is usually extrapolated from the adult data (13), moreover, the clinical and genetic features of Han Chinese pediatric PCCs/PGLs patients have never been reported.

The aim of this work was to summarize the clinical characteristics of Han Chinese pediatric PCCs/PGLs patients admitted to Xiangya Hospital, Central South University from 2006 to 2018 and to retrospectively measure the incidence of genetic mutations using wholeexome sequencing (WES).

\section{Methods}

\section{Materials and clinical data collection}

Medical records of 15 children with PCCs/PGLs who were treated and followed at Xiangya Hospital, Central South University between 2006 and 2018 were retrospectively studied. Age at diagnosis, sex, clinical presentation, laboratory evaluation, surgical treatment, and postoperative course were investigated. The diagnosis was confirmed by histopathology of surgical specimens.

\section{WES}

Qualified genomic DNA isolated from leukocytes of patients using the Dzup Kit (Dzup, Sangon Biotech Co., Ltd., Shanghai, China) was randomly fragmented using Covaris technology and the size of the library fragments was mainly distributed between 150 and $250 \mathrm{bp}$. The endrepair of DNA fragments was performed, and an "A" base was added at the 3 '-end of each strand. Adapters were then ligated to both ends of the end-repaired/A-tailed DNA fragments for amplification and sequencing. Size-selected DNA fragments were amplified using ligation-mediated PCR (LM-PCR), purified, and hybridized to the exome array for enrichment. Non-hybridized fragments were then washed out. Captured products were then circularized. Rolling circle amplification (RCA) was performed to produce DNA nanoballs (DNBs). Each resulting qualified captured library was then loaded onto a BGISEQ-500 sequencing platform, and high-throughput sequencing was performed for each captured library to ensure that each sample met the desired average sequencing coverage. Sequencing-derived raw image files were processed using BGISEQ-500 base-calling software with default parameters, and the sequence data of these patients were generated as paired-end reads. These data were defined as "raw data" and stored in the FASTQ format.

\section{Bioinformatics analysis}

Bioinformatics analysis began with the sequencing data (raw data from the BGISEQ machine). First, clean data were produced using data filtering of the raw data. All clean data of each sample were mapped to the human reference genome (GRCh37/HG19). Burrows-Wheeler Aligner (BWA) software was used to perform the alignment (14). To ensure accurate variant calling, we followed recommended best practices for variant analysis using the Genome Analysis Toolkit (GATK). Local realignment around indels and base quality score recalibration were performed using GATK $(15,16)$, with duplicate reads removed using Picard tools. The sequencing depth and coverage for these patients were calculated based on the alignments. All genomic variations including single nucleotide polymorphisms (SNPs) and indels were detected using software, such as HaplotypeCaller of GATK (v3.3.0). After that, the hardfiltering method was applied to obtain high-confidence variant calls. Then the SnpEff tool was applied to perform a series of annotations of the variants. SNPs in one of the 
known PCCs/PGLs-related genes were filtered. Mutations that had been reported as being pathogenic in the short genetic variations database SNP (dbSNP) were assessed as pathogenic. For variants that had not been reported, SIFT, PolyPhen2, MutationTaster and FATHMM scores were further used to predict their clinical significance.

\section{Results}

\section{Clinical features}

The characteristics of all patients are summarized in Table 1. Of the 15 pediatric patients, 12 were pathologically diagnosed with PCC and three with PGL. There were nine girls and six boys with a mean age of $14.9 \pm 3.01$ (range, 6-18) years. Most patients, $(80 \%, 12 / 15)$, presented with symptoms caused by excessive secretion of catecholamines, including hypertension, dizziness, headaches, and palpitations, while three presented with loin pain, convulsions, and incidental discovery, respectively. Four patients had a positive family history with a first-degree relative known to have had PCC or PGL at diagnosis, four were diagnosed with syndromic disease, and four were diagnosed with a bilateral PCC. The mean diameter of the tumors was $5.3 \pm 3.9$ (range, 1.1-20.0) $\mathrm{cm}$. Tumors were successfully resected using an adrenal-sparing approach, except for the tumor of patient PCC08 because of the large diameter $(20.0 \mathrm{~cm})$; in addition, these patients were followed for 1-12 years. During follow-up, patient PCC04 was diagnosed with a recurrent PCC with pulmonary metastasis (Figure 1). Interestingly, patient PGL01 was histologically diagnosed with pulmonary metastatic PGL 6 years after the first surgery; however, a CT scan did not show any recurrent lesion in the retroperitoneum.

\section{Case report of PCC12}

A male patient, PCC12, born in February 2005, visited Xiangya Hospital in October 2015 after experiencing episodic dizziness and sweating for 2 years. He had no family history of PCCs/PGLs. He was found to have hypertension with a blood pressure of $190 / 130 \mathrm{mmHg}$ and a peak heart rate of 141 beats/minute during the physical examination. Routine blood tests were unremarkable; however, vanillylmandelic acid (VMA) was found to be 129.0 (reference range, 5-30) $\mu \mathrm{mol} /$ day. An abdominal CT revealed a $3.2 \times 2.3 \mathrm{~cm}$ solid mass localized in the right adrenal gland (Figure 2). The adrenal tumor was surgically resected and histologically diagnosed as PCC. His DNA was sequenced in August 2017 using WES, and the mutation, $V H L$ c.193 T $>$ A was identified. PCR and Sanger sequencing of his family members did not identify this mutation. Therefore, it was considered a de novo mutation. Bioinformatics analysis was used to evaluate its function in silico (Table 2), and it was determined to be a pathogenic mutation. Subsequently, an abdominal CT scan revealed a $1.5-\mathrm{cm}$ lesion in the pancreatic body, and this was surgically removed and histologically diagnosed as a pancreatic neuroendocrine tumor (PNET) in February 2018 (Figure 2). Thus, he was clinically diagnosed with a $V H L$ syndrome according to the clinical features (17), and this was consistent with the genetic mutation.

\section{Results of WES}

To discover genetic variations in this study, we performed WES, generating $17,742.96 \mathrm{Mb}$ of raw bases on average. After removing low-quality reads, we obtained $149,714,228.5$ clean reads, on average. The mean sequencing depth of targeted regions was 149.451-fold. On average, per individual sequencing, $99.42 \%$ of targeted bases were covered by at least $1 \times$ coverage, and $97.06 \%$ of targeted bases had at least $10 \times$ coverage. On average, we identified 115,379.7 SNPs from all individuals; $97.35 \%$ were represented in the dbSNP and $93.60 \%$ were annotated in the 1,000 Genomes Project database. The number of novel SNPs was 2,471.7 on average. There were, on average, 19,641.7 indels called in all samples, of which $79.45 \%$ were represented in the dbSNP and $58.75 \%$ were annotated in the 1,000 Genomes Project database. The number of novel indels was 3,717.4, on average (Table 3).

\section{Variations in PCCs/PGLs-related genes}

A total of 182 SNPs and indels of PCCs/PGLs-related genes were identified among the patients, of which eight with a pathogenic significance were reported in the dbSNP (Table 3), one was reported in the dbSNP with conflicting interpretations of pathogenicity, and eight (VHL c. $193 \mathrm{~T}>\mathrm{A}, B R C A 2$ c.7528C $>\mathrm{A}, C D K N 2 A$ c. $49 \mathrm{C}>\mathrm{G}, K M T 2 D$ c. $6281 \mathrm{C}>\mathrm{T}, K M T 2 D$ c. $15424 \mathrm{~T}>\mathrm{C}$, ATRX c.2426A>C, SDHA c.26G>A, and GNAS c.285C>G) had not been reported previously (Table 2). Four SNPs (VHL c.193T >A, BRCA2 c.7528C >A, CDKN2A c.49C >G, and $A T R X$ c.2426A>C) were predicted to be pathogenic and one (KMT2D c. $15424 \mathrm{~T}>\mathrm{C})$ was predicted to be 


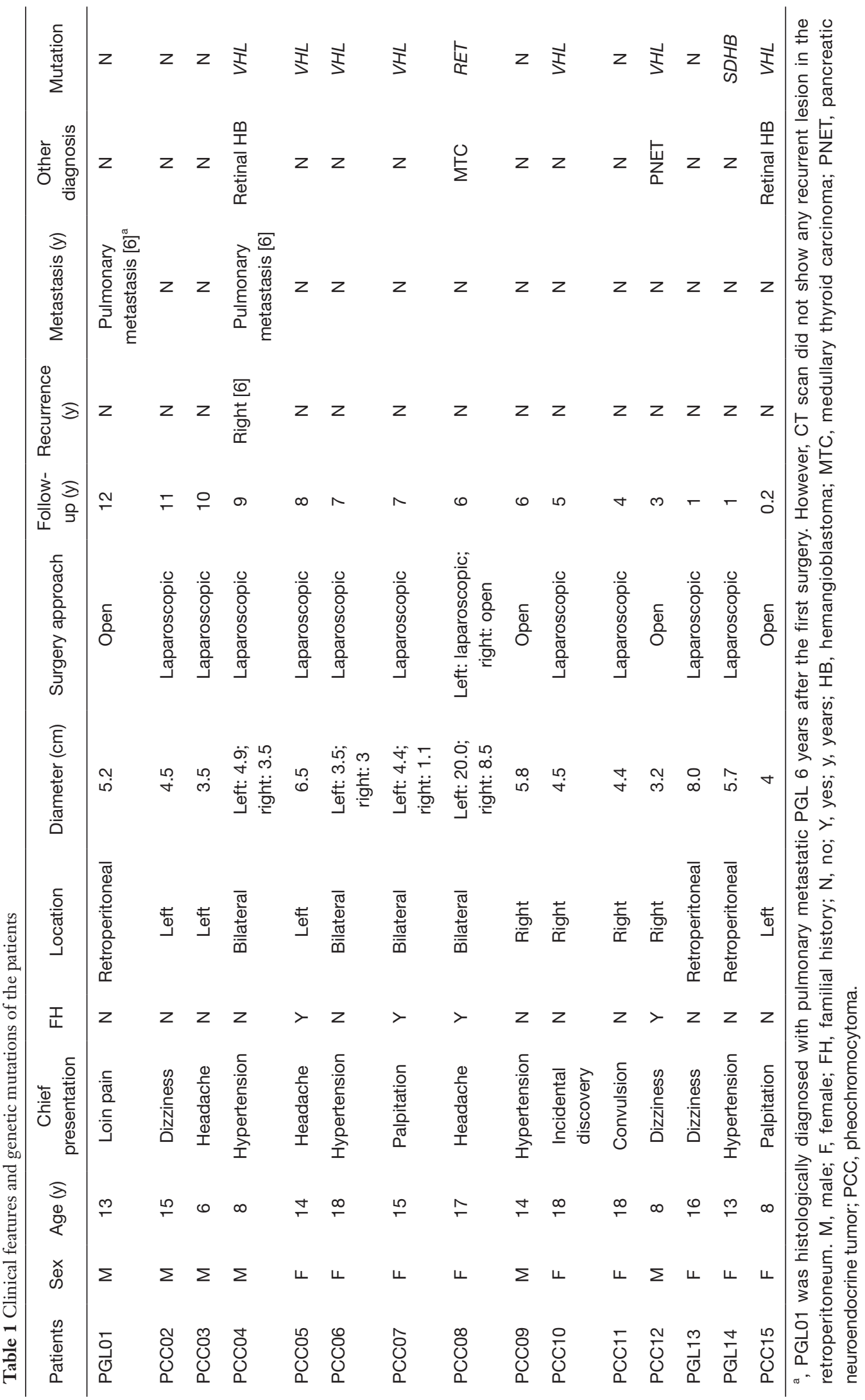



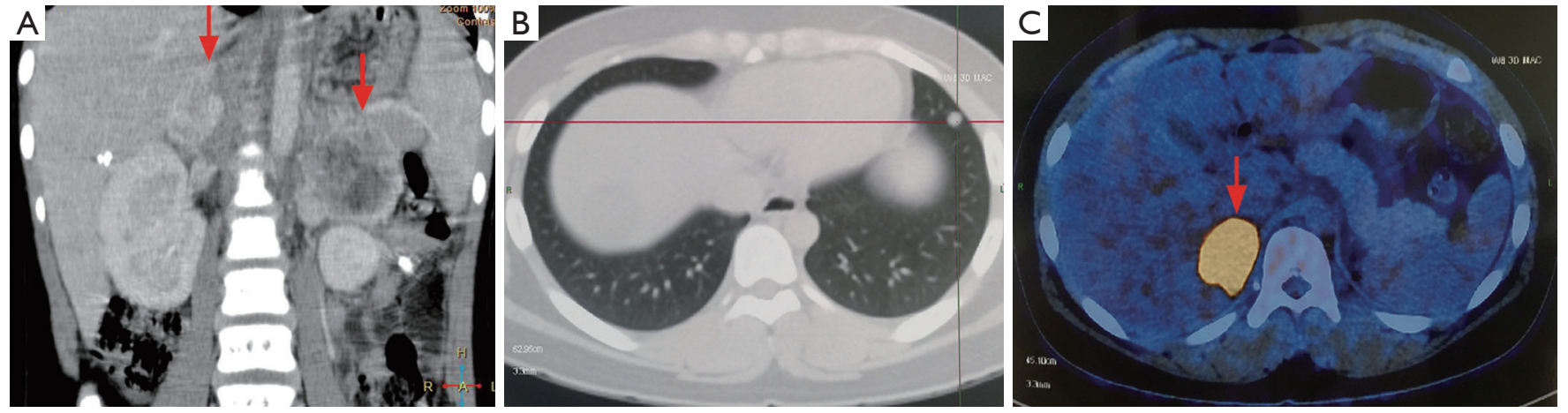

Figure 1 Medical imaging of PCC04. (A) Preoperative [2010] CT scan shows bilateral lesions of the adrenal glands (red arrow); (B,C) postoperative [2016] PET-CT scan of the whole body identified a recrudescent lesion in the right retroperitoneum and multiple metastatic pulmonary nodules (red arrow: recrudescent lesion of the right retroperitoneum; cross: one of the metastatic pulmonary nodules). PCC, pheochromocytoma.
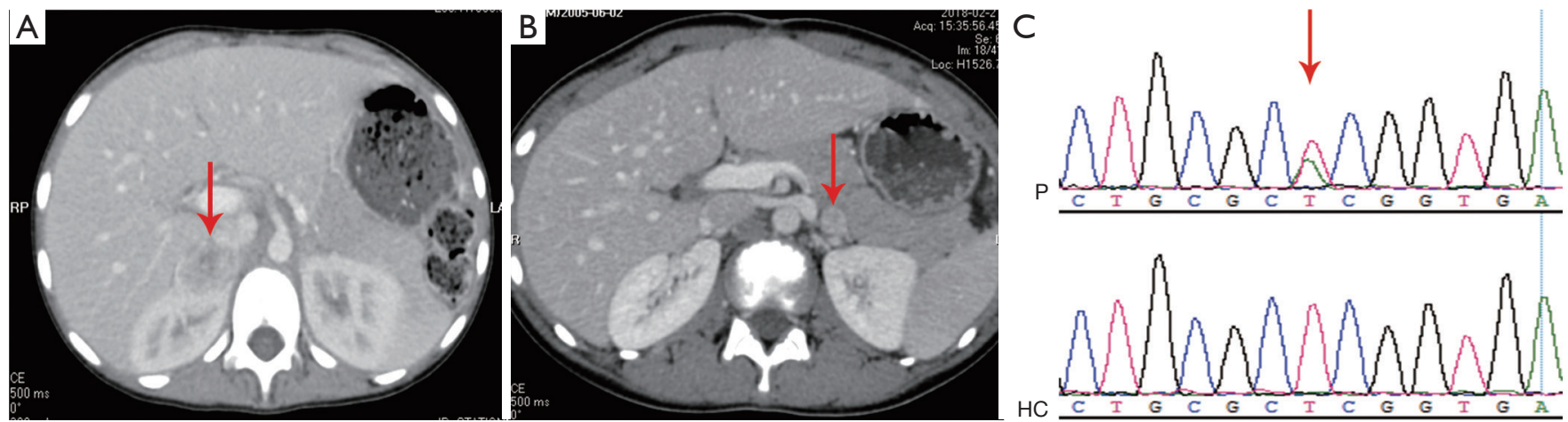

Figure 2 Clinical and genetic features of patient PCC12. (A) PCC of the right adrenal gland (arrow) discovered by a CT scan in October 2015; (B) PNET (arrow) in the pancreatic body discovered in February 2018; (C) discovery of the de novo VHL mutation, c.193T >A. PCC, pheochromocytoma; PNET, pancreatic neuroendocrine tumor.

pathogenic/likely pathogenic, according to the analysis using SIFT, PolyPhen2, MutationTaster and FATHMM prediction algorithms (Table 2). Nevertheless, except for $V H L$ c.193 $>$ A, these mutations were concluded to have an unclear significance because there were no clinical or functional data. In total, nine (60\%) patients were identified with genetic mutations (Table 3), among these mutations, seven were $V H L$, one was $R E T$, and one was $S D H B$. In addition, a de novo mutation, $V H L$ c.193 T >A, which had not been previously reported, was identified and proven to be pathogenic, according to the clinical diagnosis and bioinformatics analysis in silico.

\section{Discussion}

We have described the clinical features and genetic mutations in 15 Han Chinese pediatric patients with PCCs/PGLs in a single-center over a 10 -year period. Furthermore, the germline mutation, VHL c.193T >A, which had never been reported before, was identified and proven to be a pathogenic mutation.

Adrenal-sparing surgery is recommended for bilateral PCCs/PGLs patients by Neumann et al.; however, there is a higher risk of recurrence and metastasis (8). Four patients were diagnosed with a bilateral PCC, three of whom were carrying the $V H L$ mutation and were treated with minimally invasive adrenalectomy for the purpose of preserving adrenal function. Nevertheless, patient PCC04 was diagnosed with recurrent PCC in the right retroperitoneum. In addition, metastatic PCC was diagnosed as multiple nodes that were found in his chest using PET-CT (Figure 1). There is no doubt that adrenal 
Table 2 Mutations have not been reported and analysis in silico

\begin{tabular}{|c|c|c|c|c|c|c|c|c|c|c|}
\hline Patients & Func & Gene & Biotype & Transcript & $\begin{array}{l}\text { Codon } \\
\text { change }\end{array}$ & $\mathrm{SIFT}^{\mathrm{a}}$ & PolyPhen $2^{b}$ & MutationTaster ${ }^{c}$ & FATHMM $^{d}$ & Conclusion \\
\hline PCC01 & $\begin{array}{c}\text { Missense } \\
\text { variant }\end{array}$ & $S D H A$ & MISSENSE & $\begin{array}{c}\text { NM_004168.3:p. } \\
\text { Arg9GIn/ } \\
\text { c. } 26 G>A\end{array}$ & $\mathrm{cGg} / \mathrm{cAg}$ & 0.05 (D) & $0.003(\mathrm{~B})$ & $1.000(\mathrm{~N})$ & $-0.67(\mathrm{~T})$ & $\begin{array}{l}\text { Likely } \\
\text { benign }\end{array}$ \\
\hline PCC01 & $\begin{array}{c}\text { Missense } \\
\text { variant }\end{array}$ & GNAS & MISSENSE & $\begin{array}{c}\text { NM_080425.2:p. } \\
\text { Ser95Arg/ } \\
\text { c.285C>G }\end{array}$ & $\mathrm{agC/agG}$ & $0.23(\mathrm{~T})$ & NA & 1.000 (D) & NA & NA \\
\hline PCC01 & $\begin{array}{c}\text { Missense } \\
\text { variant }\end{array}$ & $K M T 2 D$ & MISSENSE & $\begin{array}{c}\text { NM_003482.3:p. } \\
\text { Cys5142Arg/ } \\
\text { c.15424T>C }\end{array}$ & Tgt/Cgt & 0 (D) & $0.648(P)$ & 1.000 (D) & $-1.21(\mathrm{~T})$ & $\begin{array}{c}\text { Likely } \\
\text { pathogenic }\end{array}$ \\
\hline PCC03 & $\begin{array}{c}\text { Missense } \\
\text { variant }\end{array}$ & $K M T 2 D$ & MISSENSE & $\begin{array}{c}\text { NM_003482.3:p. } \\
\text { Ala2094Val/ } \\
\text { c.6281C>T }\end{array}$ & $\mathrm{gCc} / \mathrm{gTc}$ & 0 (D) & $0.452(P)$ & $0.972(\mathrm{~N})$ & $-1.27(\mathrm{~T})$ & $\begin{array}{l}\text { Likely } \\
\text { benign }\end{array}$ \\
\hline PCC12 & $\begin{array}{c}\text { Missense } \\
\text { variant }\end{array}$ & ATRX & MISSENSE & $\begin{array}{c}\text { NM_000489.4:p. } \\
\text { GIn809Pro/ } \\
\text { c.2426A>C }\end{array}$ & $\mathrm{cAa} / \mathrm{cCa}$ & $0.03(\mathrm{D})$ & $0.091(\mathrm{~B})$ & 1.000 (D) & $-3.13(\mathrm{D})$ & Pathogenic \\
\hline PCC14 & $\begin{array}{c}\text { Missense } \\
\text { variant }\end{array}$ & BRCA2 & MISSENSE & $\begin{array}{c}\text { NM_000059.3:p. } \\
\text { Leu2510Met/ } \\
\text { c.7528C>A }\end{array}$ & $\mathrm{Ctg} / \mathrm{Atg}$ & 0.0 (D) & $1.0(D)$ & $0.999483(\mathrm{D})$ & $-2.84(\mathrm{D})$ & Pathogenic \\
\hline PCC14 & $\begin{array}{c}\text { Missense } \\
\text { variant }\end{array}$ & CDKN2A & MISSENSE & $\begin{array}{c}\text { NM_058195.3:p. } \\
\text { Pro17Ala/ } \\
\text { c.49C>G }\end{array}$ & $\mathrm{Ccg} / \mathrm{Gcg}$ & 0.04 (D) & $0.856(P)$ & 1 (D) & $-1.89(\mathrm{D})$ & Pathogenic \\
\hline
\end{tabular}

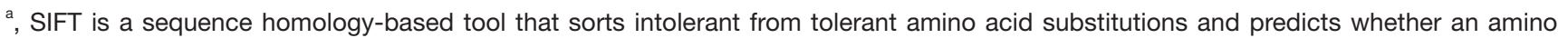
acid substitution in a protein will have a phenotypic effect. Range, $[0,1]$. The amino acid substitution is predicted damaging (D) is the score is $\leq 0.05$, and tolerated $(T)$ if the score is $>0.05$; ${ }^{b}$, PolyPhen2 is an automatic tool for prediction of possible impact of an amino acid substitution on the structure and function of a human protein. Range, [0,1]. The more the score is close to 1 , the more damage the mutation is. A mutation is also appraised qualitatively, as benign $(B)$, possibly damaging $(P)$, or probably damaging (D) based on pairs of false positive rate thresholds, optimized separately for each model; ${ }^{\mathrm{C}}$, MutationTaster is an in silico predictive algorithm that predicts an alteration as one of four possible types: disease-causing (D), disease-causing automatic (A), polymorphism (N), or polymorphism automatic $(\mathrm{P})$. Range, $[0,1]$. MutationTaster reflects the probability that each nucleotide belongs to a conserved element, the higher the values of PhyloP, the more probable the nucleotide is conserved; ${ }^{d}$, FATHMM is an in silico predictive algorithm of the functional consequences. Predictions are given as $P$ values in the range $[0,1]$; values above 0.5 are predicted to be deleterious (D), while those below 0.5 are predicted to be neutral or benign (B). PCC, pheochromocytoma; NA, not available.

preservation is key in children, and this helps to maximize their life expectancy. However, adrenal-preserving surgery can also augment the risk of recurrence and metastasis, which is of great significance for pediatric patients as $50 \%$ of this population is reported to have metastatic disease (13). More importantly, the prognosis of patients with recurrent and metastatic PCCs/PGLs is poor, with limited treatment options (18). Knowing this, we cannot directly correlate the surgical approach and experiences of adult to pediatric patients with a bilateral PCC and further research should be done in terms of pediatric PCCs/PGLs for determining better treatments in this population. Furthermore, genetic testing should be performed prior to surgery for the purpose of identifying mutations.

Four patients $(26.7 \%)$ in our study were also diagnosed with other syndromic tumors; two were retinal hemangioblastomas (RHBs), one was a PNET, and one was a medullary thyroid carcinoma (MTC). It is well documented that all patients carrying a germline RET mutation suffer from MTC and 50\% will contract PCCs/ 
Table 3 Result of WES and variations of the patients

\begin{tabular}{|c|c|c|c|c|c|c|c|c|}
\hline Patients & $\begin{array}{l}\text { Number of } \\
\text { clean reads }\end{array}$ & $\begin{array}{c}\text { Average } \\
\text { sequencing } \\
\text { depth }\end{array}$ & SNPS & Novel SNPs & Indels & Novel indels & $\begin{array}{c}\text { Number of variants } \\
\text { in PPGL related } \\
\text { genes }\end{array}$ & $\begin{array}{c}\text { Variants with } \\
\text { pathogenic } \\
\text { significant }\end{array}$ \\
\hline PCC02 & $117,007,224$ & 136.35 & 115,762 & 2,440 & 18,508 & 3,127 & 15 & $\mathrm{~N}$ \\
\hline PCC03 & $119,444,828$ & 133.54 & 120,050 & 2,307 & 20,880 & 3,677 & 11 & $\mathrm{~N}$ \\
\hline PCC05 & $114,988,366$ & 130.68 & 118,279 & 2,339 & 20,220 & 3,505 & 10 & $V H L$ \\
\hline PCC06 & $355,560,714$ & 229.87 & 110,516 & 1,960 & 19,348 & 4,110 & 12 & $V H L$ \\
\hline PCC07 & $128,266,856$ & 151.61 & 118,425 & 2,331 & 20,655 & 3,675 & 12 & $V H L$ \\
\hline PCC08 & $218,976,116$ & 164.14 & 106,803 & 1,672 & 18,224 & 3,578 & 14 & $R E T$ \\
\hline PCC11 & $149,253,846$ & 175.25 & 120,490 & 2,073 & 20,819 & 3,830 & 9 & $\mathrm{~N}$ \\
\hline PCC12 & $97,378,262$ & 118.02 & 113,869 & 2,958 & 18,728 & 3,542 & 16 & $V H L$ \\
\hline PGL13 & $149,253,846$ & 175.25 & 120,490 & 2,073 & 20,819 & 3,830 & 9 & $\mathrm{~N}$ \\
\hline PGL14 & $112,001,240$ & 136.06 & 116,632 & 2,260 & 19,697 & 3,316 & 13 & $S D H B$ \\
\hline PCC15 & $221,074,986$ & 203.37 & 99,155 & 1,623 & 16,624 & 3,399 & 11 & $V H L$ \\
\hline Average & $149,714,228.5$ & 149.451 & $115,379.7$ & $2,471.7$ & $19,641.7$ & $3,717.4$ & 12.1 & - \\
\hline
\end{tabular}

PPGL, which means PCC/PGL in this field. WES, whole-exome sequencing; PGL, paraganglioma; PCC, pheochromocytoma; SNP, single nucleotide polymorphism.

PGLs. Thus, it is strongly recommended that PCCs/PGLs patients who carry a RET mutation undergo prophylactic thyroidectomy (19). Notably, over $90 \%$ of patients who carry a $V H L$ mutation will manifest a $V H L$ syndromerelated disease by the age of 65 years, while PCC only occurs in $20 \%$ of $V H L$ patients (20). Germline mutations, including $N F 1$ and $S D H x$ can also cause other syndromic diseases in addition to PCCs/PGLs (10). Therefore, a genetic diagnosis for patients with PCCs/PGLs may lead to early detection of other syndromic diseases, treatment modifications, and routine surveillance for common syndrome-related manifestations.

A male patient, PCC12, presented to our hospital in 2015 and was found to carry the $V H L$ c.193T $>$ A mutation, which had never been previously reported. This variant was predicted to have pathogenic significance for several reasons. First, the analyses based on the prediction algorithms SIFT, PolyPhen2, MutationTaster, and FATHMM provided bioinformatic proof. Second, mutations at the same site, VHL c.193T >C and VHL c.193T>G, were reported previously to be pathogenic and likely pathogenic mutations, respectively, which indicates that $V H L$ c.193T $>$ A could be a pathogenic variant according to the "American College of Medical Genetics and Genomic Guidelines" (evidence PM5) (21). In addition, evidence of PM2, PM1, and PS2 could also be applied to the mutations found in the patient. Although well-established functional studies are lacking, we strongly suggest that the mutation $V H L$ c.193T >A has pathogenic significance. In addition, clinical examinations were recommended for this patient and PNET was detected using CT and successfully resected, this highlights the importance and necessity of genetic screening and targeted follow-up and examinations according to the genetic mutation.

In our study, 9 of $15(60 \%)$ patients were identified as carrying germline genetic mutations. Of the patients who had no family history of PCCs/PGLs, $45.5 \%$ had germline mutations. In addition, $26.7 \%$ of the patients in our study were diagnosed with bilateral PCCs or syndromic tumors plus PCCs that involved genetic mutations. It has been 
reported that positive genetic mutations are associated with bilateral or multifocal PCCs, gene-related diseases, and metastatic PPCs/PGLs (22). However, in clinical practice, only a fraction of patients are genetically tested. Dias Pereira et al. suggested that all children, adolescents, and young adults with PCC or PGL should undergo genetic testing (23). We do believe that and our work strengthened the importance of genetic testing in pediatric PCCs/PGLs patients regardless of a lack of family history because of the high percentage of germline genetic mutations and generelated morbidity.

Our study had limitations. We approached the importance of genetic consulting for pediatric patients with PCCs/PGLs, especially in the Han Chinese population, which has not been previously reported. However, only 15 patients from a single-center were included, regardless of the scarceness of these types of diseases. Four mutations that have not been reported, BRCA2 c.7528C>A, CDKN2A c.49C>G, ATRX c.2426A>C, and KMT2D c. $15424 \mathrm{~T}>\mathrm{C}$, were identified using WES. Although bioinformatics analysis suggested that they may be pathogenic, we did not consider these mutations to be the cause of disease in our patients because there is little known about these genes and their relationship to the morbidity of PCCs/PGLs. Furthermore, there was a lack of functional or clinical evidence associated with these mutations found in our study.

In conclusion, we have provided the clinical features of 15 Han Chinese pediatric PCCs/PGLs patients from a single-center over a 10 -year period. Among the 15 patients, $60 \%$ were identified as carrying germline genetic mutations, four were diagnosed with bilateral PCCs, and four were diagnosed with other syndromic tumors in addition to their PCCs, which indicates that it is imperative that all pediatric PCCs/PGLs patients undergo genetic testing and are managed and followed according to the results of their genetic tests. Furthermore, this is the first report of the pathogenic mutation, VHL c.193T $>$ A.

\section{Acknowledgments}

We thank Mark Abramovitz, PhD, from Liwen Bianji, Edanz Group China (www.liwenbianji.cn/ac), for editing the English text of a draft of this manuscript.

Funding: This work was supported by the National Natural Science Foundation of China (No. 81400773), Innovation-Driven Project of Central South University (No. 020CX046), Funds for the Shenghua Yuying talents program of Central South University and the Fundamental
Research and Funds for the Graduate Students of Central South University (No. 2017zzts901).

\section{Footnote}

Conflicts of Interest: All authors have completed the ICMJE uniform disclosure form (available at http://dx.doi. org/10.21037/tau.2020.02.14). The authors have no conflicts of interest to declare.

Ethical Statement: The authors are accountable for all aspects of the work in ensuring that questions related to the accuracy or integrity of any part of the work are appropriately investigated and resolved. The ethics committee of Xiangya Hospital (201905133) approved the study, and written informed consent was obtained from all patients and their parents.

Open Access Statement: This is an Open Access article distributed in accordance with the Creative Commons Attribution-NonCommercial-NoDerivs 4.0 International License (CC BY-NC-ND 4.0), which permits the noncommercial replication and distribution of the article with the strict proviso that no changes or edits are made and the original work is properly cited (including links to both the formal publication through the relevant DOI and the license). See: https://creativecommons.org/licenses/by-nc$\mathrm{nd} / 4.0 /$.

\section{References}

1. Waguespack SG, Rich T, Grubbs E, et al. A current review of the etiology, diagnosis, and treatment of pediatric pheochromocytoma and paraganglioma. J Clin Endocrinol Metab 2010;95:2023-37.

2. Ross JH. Pheochromocytoma. Special considerations in children. Urol Clin North Am 2000;27:393-402.

3. Dahia PL. Pheochromocytoma and paraganglioma pathogenesis: learning from genetic heterogeneity. Nat Rev Cancer 2014;14:108-19.

4. Pillai S, Gopalan V, Smith RA, et al. Updates on the genetics and the clinical impacts on phaeochromocytoma and paraganglioma in the new era. Crit Rev Oncol Hematol 2016;100:190-208.

5. De Krijger RR, Petri BJ, Van Nederveen FH, et al. Frequent genetic changes in childhood pheochromocytomas. Ann N Y Acad Sci 2006;1073:166-76.

6. Bausch B, Wellner U, Bausch D, et al. Long-term 
prognosis of patients with pediatric pheochromocytoma. Endocr Relat Cancer 2013;21:17-25.

7. PDQ Adult Treatment Editorial Board. Pheochromocytoma and Paraganglioma Treatment (PDQ®): Health Professional Version. PDQ Cancer Information Summaries. Bethesda: National Cancer Institute (US), 2002.

8. Neumann HPH, Tsoy U, Bancos I, et al. Comparison of pheochromocytoma-specific morbidity and mortality among adults with bilateral pheochromocytomas undergoing total adrenalectomy vs cortical-sparing adrenalectomy. JAMA Netw Open 2019;2:e198898.

9. Liu P, Li M, Guan X, et al. Clinical syndromes and genetic screening strategies of pheochromocytoma and paraganglioma. J Kidney Cancer VHL 2018;5:14-22.

10. Pham TH, Moir C, Thompson GB, et al. Pheochromocytoma and paraganglioma in children: a review of medical and surgical management at a tertiary care center. Pediatrics 2006;118:1109-17.

11. Osman Y, Hussein N, Sarhan O, et al. Surgical analysis of pediatric and adolescent sporadic pheochromocytoma: single center experience. Int Urol Nephrol 2011;43:1019-24.

12. Mishra A, Mehrotra PK, Agarwal G, et al. Pediatric and adolescent pheochromocytoma: clinical presentation and outcome of surgery. Indian Pediatr 2014;51:299-302.

13. Peard L, Cost NG, Saltzman AF. Pediatric pheochromocytoma: current status of diagnostic imaging and treatment procedures. Curr Opin Urol 2019;29:493-9.

14. Li H, Durbin R. Fast and accurate long-read alignment with Burrows-Wheeler transform. Bioinformatics 2010;26:589-95.

Cite this article as: Li M, Wang C, Liu P, Qi L, Chen X, Fan B, Zhang X, Zhang B, Xiao Q, Yu A, Liu L. Clinical and genetic features of pediatric PCCs/PGLs patients: a single-center experience in China. Transl Androl Urol 2020;9(2):267-275. doi: 10.21037/tau.2020.02.14
15. DePristo MA, Banks E, Poplin R, et al. A framework for variation discovery and genotyping using next-generation DNA sequencing data. Nat Genet 2011;43:491-8.

16. McKenna A, Hanna M, Banks E, et al. The Genome Analysis Toolkit: a MapReduce framework for analyzing next-generation DNA sequencing data. Genome Res 2010;20:1297-303.

17. Lonser RR, Glenn GM, Walther M, et al. von HippelLindau disease. Lancet 2003;361:2059-67.

18. Jimenez C, Rohren E, Habra MA, et al. Current and future treatments for malignant pheochromocytoma and sympathetic paraganglioma. Curr Oncol Rep 2013;15:356-71.

19. Pacheco MC. Multiple endocrine neoplasia: a genetically diverse group of familial tumor syndromes. J Pediatr Genet 2016;5:89-97.

20. Varshney N, Kebede AA, Owusu-Dapaah H, et al. A review of von Hippel-Lindau syndrome. J Kidney Cancer VHL 2017;4:20-9.

21. Richards S, Aziz N, Bale S, et al. Standards and guidelines for the interpretation of sequence variants: a joint consensus recommendation of the American College of Medical Genetics and Genomics and the Association for Molecular Pathology. Genet Med 2015;17:405-24.

22. Lenders JW, Duh QY, Eisenhofer G, et al. Pheochromocytoma and paraganglioma: an endocrine society clinical practice guideline. J Clin Endocrinol Metab 2014;99:1915-42.

23. Dias Pereira B, Nunes da Silva T, Bernardo AT, et al. A clinical roadmap to investigate the genetic basis of pediatric pheochromocytoma: which genes should physicians think about? Int J Endocrinol 2018;2018:8470642. 